\title{
Editorial
}

\section{Nanomaterials for Thermoelectrics}

\author{
Hyung-Ho Park, ${ }^{1}$ Won-Seon Seo, ${ }^{2}$ Jin-Sang Kim, ${ }^{3}$ Chan Park, ${ }^{4}$ and Jung-Kun Lee \\ ${ }^{1}$ Department of Material Science and Engineering, Yonsei University, Seoul, Republic of Korea \\ ${ }^{2}$ Environment-Energy Ceramics, Korea Institute of Ceramic Engineering, Seoul, Republic of Korea \\ ${ }^{3}$ Electronic Materials Center, Korea Institute of Science and Technology, Seoul, Republic of Korea \\ ${ }^{4}$ Department of Material Science and Engineering, Seoul National University, Seoul, Republic of Korea \\ ${ }^{5}$ Department of Mechanical Engineering and Materials Science, University of Pittsburgh, Pittsburgh, PA, USA
}

Correspondence should be addressed to Hyung-Ho Park; hhpark@yonsei.ac.kr

Received 15 December 2013; Accepted 15 December 2013; Published 19 January 2014

Copyright (C) 2014 Hyung-Ho Park et al. This is an open access article distributed under the Creative Commons Attribution License, which permits unrestricted use, distribution, and reproduction in any medium, provided the original work is properly cited.

The production and use of fossil fuels raise environmental concerns. A global movement toward the generation of renewable energy is therefore underway to help meet increased energy needs. Among various green energy technologies, thermoelectric power generation has attracted increasing attention. Thermoelectric generation refers to the direct conversion of waste heat into useful electricity. Because of a nonpolluting and renewable technique, it is a suitable candidate for future energy conversion. High electrical conductivity, low thermal conductivity, and the high Seebeck coefficient are required for thermoelectric materials. However, it is difficult to control these factors individually because thermal conductivity is generally proportional to electrical conductivity. One way to disrupt this relationship is to control the microstructure on a nanoscale. By adopting a new design concept of phonon-glass electron-crystal, nanostructure could be adopted to optimize thermoelectric figure of merit. The goal of this special issue was to exhibit recent developments in the nanomaterials for application in thermoelectrics, so as to plot a picture of (1) the current state of the field and (2) the opportunities for future research related to nanomaterials for thermoelectrics.

A total of 10 articles are presented in the current issue and they are all research papers. They involve nanocomposite formation for thermoelectrics, nanoparticle dispersion in thermoelectric matrix, minimization of lattice thermal conductivity by formation of dense pore structure, mesoporous structured thermoelectrics, and microstructural control of thermoelectric materials. The effects of porosity, doping, mechanical deformation, and microstructure on the thermoelectric properties were discussed.
We are pleased to see the progress in a relation between microstructural control and the properties of thermoelectric materials. We hope that this special issue will contribute to the enhancement of the thermoelectric properties, economically feasible thermoelectric device technologies, and also their wide use.

\section{Acknowledgments}

The editors gratefully thank the authors for their contributions to this special issue and the reviewers for their constructive comments and dedication. 

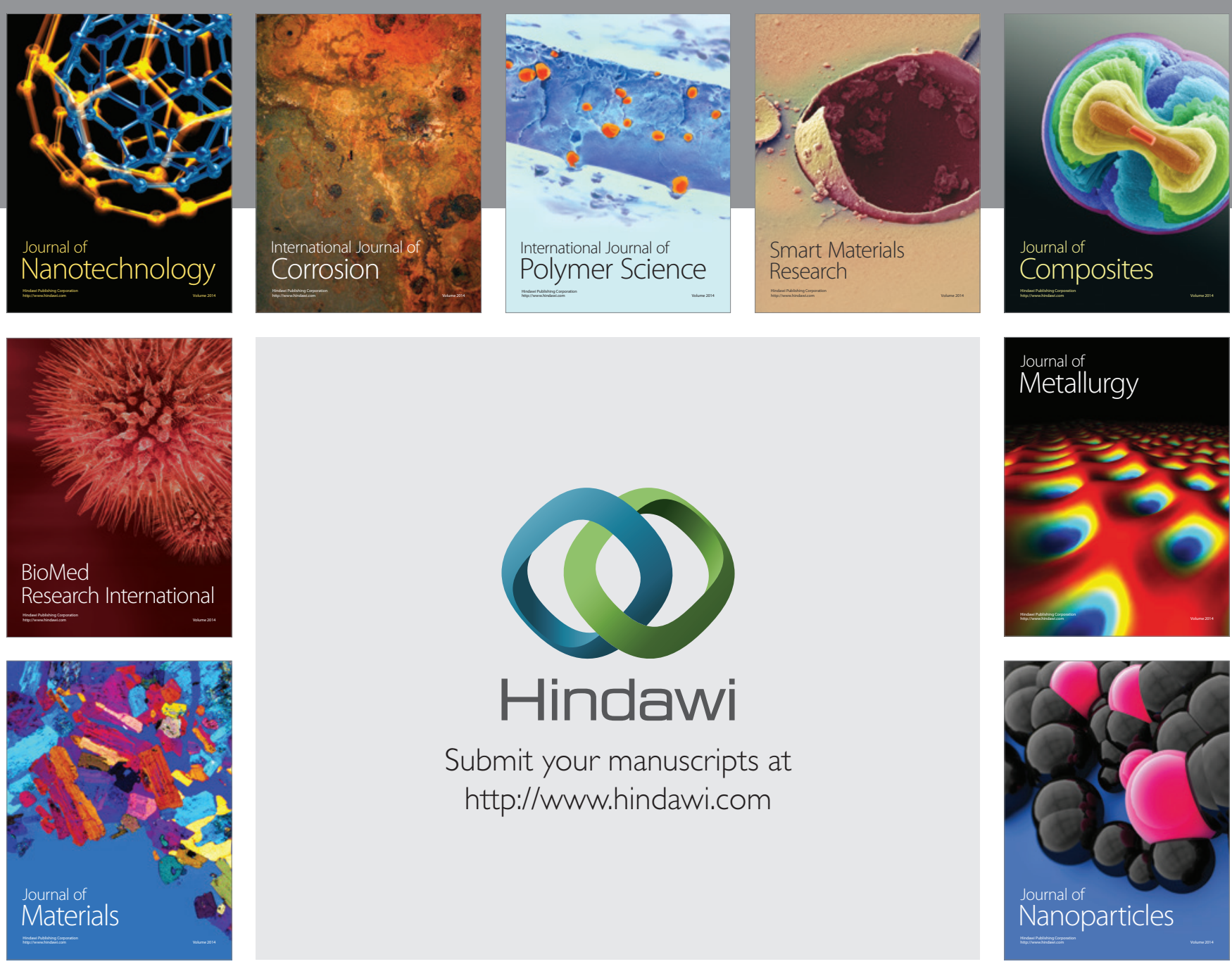

Submit your manuscripts at http://www.hindawi.com
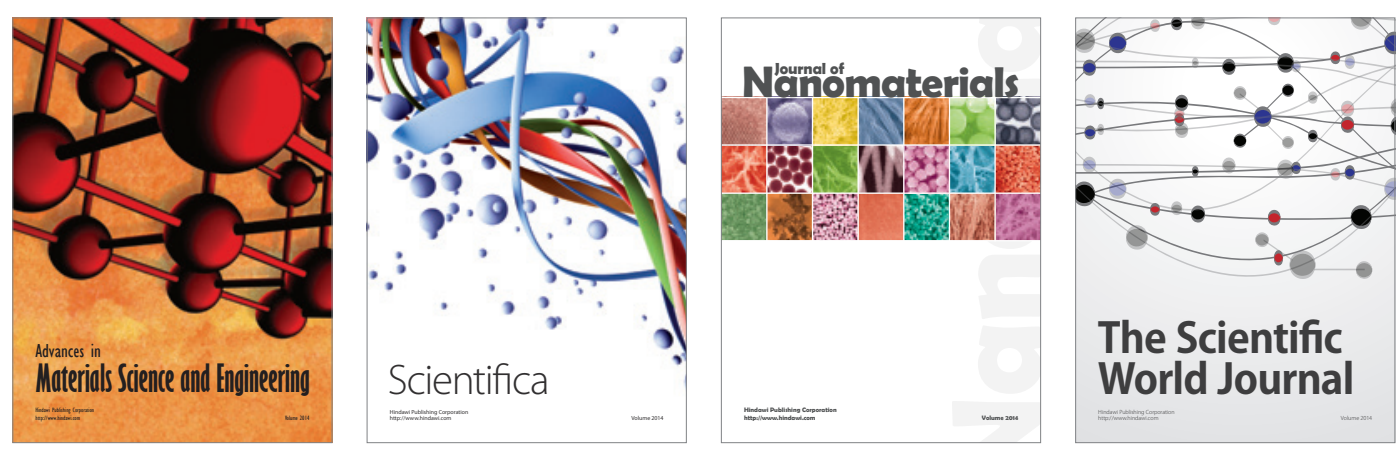

\section{The Scientific World Journal}
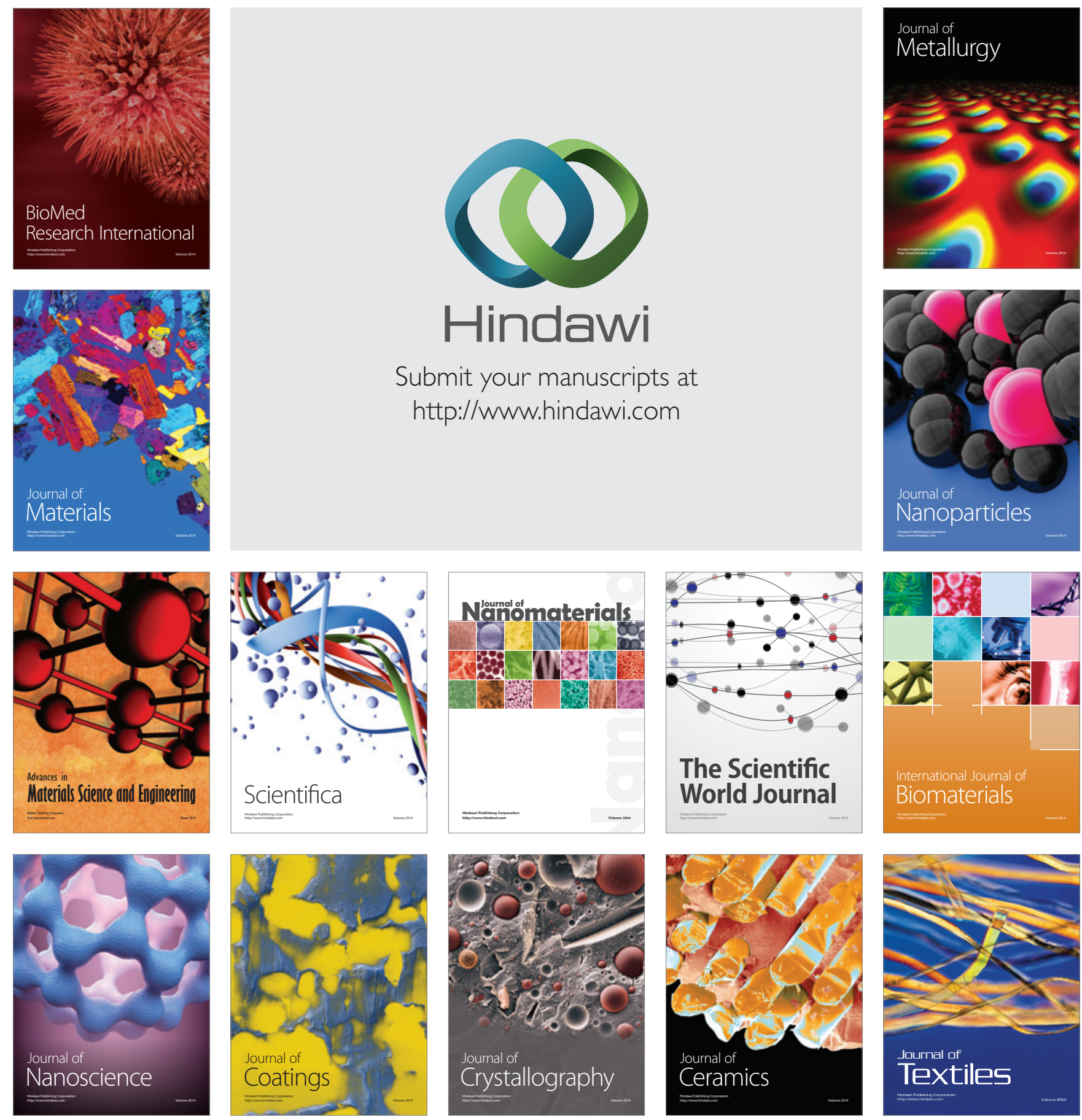